\title{
Cluster structure and Coulomb shift in two-center mirror systems
}

\author{
M. Nakao ${ }^{1}$, H. Umehara ${ }^{1}$, S. Sonoda ${ }^{1}$, S. Ebata ${ }^{2}$, and M. Ito ${ }^{1, \star}$ \\ ${ }^{1}$ Department of Pure and Applied Physics, Kansai University, 3-3-35 Yamatecho, Suita, Osaka 564-8680, Japan. \\ ${ }^{2}$ Nuclear Reaction Data Center, Faculty of Science, Hokkaido University, Sapporo 060-0810, Japan.
}

\begin{abstract}
The $\alpha+{ }^{14} \mathrm{C}$ elastic scattering and the nuclear structure of its compound systems, ${ }^{18} \mathrm{O}=\alpha+{ }^{14} \mathrm{C}$, are analyzed on the basis of the semi-microscopic model. The $\alpha+{ }^{14} \mathrm{C}$ interaction potential is constructed from the double folding (DF) model with the effective nucleon-nucleon interaction of the density-dependent Michigan 3-range Yukawa. The DF potential is applied to the $\alpha+{ }^{14} \mathrm{C}$ elastic scattering in the energy range of $E_{\alpha} / A_{\alpha}=5.5$ $\sim 8.8 \mathrm{MeV}$, and the observed differential cross sections are reasonably reproduced. The energy spectra of ${ }^{18} \mathrm{O}$ are calculated by employing the orthogonality condition model (OCM) plus the absorbing boundary condition $(\mathrm{ABC})$. The $\mathrm{OCM}+\mathrm{ABC}$ calculation predicts the formation of the $0^{+}$resonance around $E=3 \mathrm{MeV}$ with respect to the $\alpha$ threshold, which seems to correspond to the $0_{4}^{+}$resonance identified in the recent experiment. We also apply the $\mathrm{OCM}+\mathrm{ABC}$ calculation to the mirror system, such as ${ }^{18} \mathrm{Ne}=\alpha+{ }^{14} \mathrm{O}$, and the Coulomb shift of ${ }^{18} \mathrm{O}-{ }^{18} \mathrm{Ne}$ is evaluated. We have found that the Coulomb shift is clearly reduced in the excited $0^{+}$state due to the development of the $\alpha$ cluster structure. This result strongly supports that the Coulomb shift is a candidate of new probe to identify the clustering phenomena.
\end{abstract}

\section{Introduction}

$\alpha$ particle is a building block in constructing the intrinsic structures of atomic nuclei because of its stable and inert structure. The so-called $\alpha$ cluster structure appears in the excited states of light mass system, and the structures have been extensively discussed in the $N=Z$ system along the stability line [1]. For example, the structure of ${ }^{8} \mathrm{Be}=2 \alpha$, ${ }^{12} \mathrm{C}=3 \alpha,{ }^{16} \mathrm{O}=\alpha+{ }^{12} \mathrm{C}$ and ${ }^{20} \mathrm{Ne}=\alpha+{ }^{16} \mathrm{O}$ are the typical examples of the $\alpha$ cluster structure. The studies of the $\alpha$ cluster structures in the $N=Z$ system is still developed. For example, in recent studies, the multi- $\alpha$ structures, such as ${ }^{12} \mathrm{C}=3 \alpha[2],{ }^{16} \mathrm{O}=4 \alpha[2,3]$ and ${ }^{20} \mathrm{Ne}=5 \alpha[2]$, are extensively discussed, and they are interpreted in terms of the "nuclear $\alpha$-particle condensation state" in a bosonic mean field [4].

The research on the $\alpha$ cluster structures are extended to nuclei which are not on the non-stability line with $N \neq Z$. In the $N \neq Z$ systems, the cluster degrees of freedom are also important, and fruitful structures are widely discussed. In particular, the $\alpha$ cluster structures are extensively investigated in neutron excess $(N>Z)$ systems [57]. In neutron excess systems, the valence neutrons play a glue-like role among the cluster cores, which is associated with covalent electrons in molecular systems, and the various chemical-bonding structures are generated. We can see the typical example of such the chemical-bonding structure in Be isotopes, in which excess neutrons form the covalent orbit around the two center cores of ${ }^{8} \mathrm{Be}=$ $\alpha+\alpha$. In ${ }^{10,12} \mathrm{Be}$, for example, the prominent structural changes among the chemical bonding structure, such as

\footnotetext{
^e-mail: itomk@kansai-u.ac.jp
}

the covalent, atomic and ionic configurations, occurs in the unbound continuum region, and they are strongly excited by the $\alpha+{ }^{6,8} \mathrm{He}$ resonant scattering [5].

An interesting viewpoint for the cluster structures in the $N \neq Z$ systems is the Coulomb shift for the mirror systems, which are obtained by replacing neutrons to protons. Generally speaking, the energy levels in the protonrich side $(N<Z)$ shift to the higher energy region than the levels in the neutron-rich side $(N>Z)$ because of the difference in the Coulomb interaction. One of the famous phenomena relevant to the Coulomb shift is the so-called "Thomas-Ehrman Shift (TES)", which was discussed in the mirror system of ${ }^{17} \mathrm{O}={ }^{16} \mathrm{O}+n$ and ${ }^{17} \mathrm{~F}={ }^{16} \mathrm{O}+p[8]$. If the valence nucleon occupies the $\mathrm{S}$-wave orbit, the wave function is spatially extended in comparison to the distribution of the non S-wave orbit because of lacking the centrifugal barrier in the S-wave single particle motion. Thus, the effect of the Coulomb interaction is more suppressed for the S-wave state than that for the non S-wave state. As a result of this suppressed Coulomb effect, the excitation energy of the $1 / 2^{+}$state in the proton-rich ${ }^{17} \mathrm{~F}$ seems to be lower than that in the neutron-rich ${ }^{17} \mathrm{O}[8]$.

TES is a kind of anomalous phenomena, which is induced by the S-wave configuration in the nucleon orbit. Such the S-wave configuration can also be seen in the cluster state, which often appears in the excited $0^{+}$state of the even-nuclear systems. In the cluster structure, generally speaking, the relative wave function of the $\alpha$ particle and the residual nuclei are weakly coupled, and they mainly perform the S-wave relative motion. Thus, the Coulomb interaction is not so effective in the case that the cluster 
structures are strongly developed. In this situation, the reduction of the Coulomb shift, which is similar to the phenomenon discussed in ${ }^{17} \mathrm{O}-{ }^{17} \mathrm{~F}$, is expected to be observed. Therefore, the reduced Coulomb shift is a new candidate to identify the cluster degrees of freedom in the excited $0^{+}$state. In fact, the reduction of the Coulomb shift due to the cluster formation is demonstrated in the mirror system of ${ }^{10} \mathrm{Be}-{ }^{10} \mathrm{C}$ [9].

In the present report, we extend the analysis of the Coulomb shift studied in ${ }^{10} \mathrm{Be}-{ }^{10} \mathrm{C}$ to the heavier systems, such as ${ }^{18} \mathrm{O}-{ }^{18} \mathrm{Ne}$, which are handled by the cluster models of $\alpha+{ }^{14} \mathrm{C}$ and $\alpha+{ }^{14} \mathrm{O}$, respectively. In ${ }^{18} \mathrm{O}$, the $\alpha+{ }^{14} \mathrm{C}$ structure has already been discussed by the full microscopic model, in which the anti-symmetrization among all the nucleons are completely taken into account $[10,11]$. However, the energy levels of the excited $0^{+}$ states, which have the well developed $\alpha+{ }^{14} \mathrm{C}$ structure, is much higher than the observed $0^{+}$levels and hence, the direct comparison of the theory with the experiment is still difficult. This overestimation of the excitation energies is a peculiar problem in the full microscopic models, which are difficult to reproduce the $\alpha$ threshold and the energy levels simultaneously.

In order to overcome the threshold problem, it is important to introduce the semi-microscopic model, which is free from the reproduction of the threshold energies theoretically. The orthogonality condition model (OCM) is very useful and powerful model as such the semimicroscopic approach [12]. In the OCM calculation, the functional space allowed by the Pauli's exclusion principle is constructed by solving the eigenvalue problem of the norm kernels, which are the overlap functions of the cluster wave function with the full anti-symmetrization. After constructing the Pauli allowed states, we can apply the simple potential model in solving the cluster relative motion.

In the present report, we apply the OCM to the mirror cluster-systems of ${ }^{18} \mathrm{O}=\alpha+{ }^{14} \mathrm{C}$ and ${ }^{18} \mathrm{Ne}=\alpha+{ }^{14} \mathrm{O}$ and discuss the Coulomb shift in connection to the cluster formation. The cluster interaction is constructed by the double folding (DF) model, and the validity of the DF potential is checked by the analysis of the $\alpha+{ }^{14} \mathrm{C}$ elastic scattering. The DF potential, which is used for the scattering calculation, is applied to the OCM calculation with the $\alpha+{ }^{14} \mathrm{C}$ and $\alpha+{ }^{14} \mathrm{O}$ structures. The cluster states, which appear as the resonant state above the $\alpha$ threshold, are identified by imposing the absorbing boundary condition $(\mathrm{ABC})$ [21]. In the calculation of $\mathrm{OCM}+\mathrm{ABC}$, we formulate a new computational technique to handle the norm kernel on the basis of the simple numerical calculation because the evaluation of the norm kernel in OCM is complicated for general cluster systems of $N \neq Z$.

The organization of this article is the following. In Sec. 2, the construction of the DF potential, and the new OCM framework plus the ABC method are briefly explained. The results of the numerical calculations are shown in Sec. 3. First, the application of the DF potential to the $\alpha+{ }^{14} \mathrm{C}$ elastic scattering is presented. Secondly, the energy calculation for ${ }^{18} \mathrm{O}=\alpha+{ }^{14} \mathrm{C}$ by using OCM $+\mathrm{ABC}$ is performed. Finally, the Coulomb shift of ${ }^{18} \mathrm{O}$ and ${ }^{18} \mathrm{Ne}$ is discussed in connection to the development of the $\alpha$ clusters. The final section is devoted to the summary and discussion.

\section{Framework}

In our analyses, there are two steps in the calculations; first, we solve the scattering problem of the $\alpha+{ }^{14} \mathrm{C}$ system by employing the double folding (DF) potential, and the validity of the DF potential is checked. Secondly, the energy spectra of the respective compound system, ${ }^{18} \mathrm{O}=\alpha+{ }^{14} \mathrm{C}$, is calculated by the orthogonality condition model (OCM) under the absorbing boundary condition $(\mathrm{ABC})$. In the following sections, we explain our models for solving the scattering and structure problems.

\section{$2.1 \alpha-{ }^{14} \mathrm{C}$ Double folding potential}

We calculate the differential cross sections of an $\alpha$ particle scattered by ${ }^{14} \mathrm{C}$ in the formulation of the microscopic nuclear interaction. The nuclear potential of $\alpha$ and ${ }^{14} \mathrm{C}$ is calculated from the double folding (DF) model [13], which is symbolically written as a function of the $\alpha-{ }^{14} \mathrm{C}$ relative coordinate $\mathbf{R}$,

$$
U_{\mathrm{DF}}(\mathbf{R})=\iint \rho_{\alpha}\left(\mathbf{r}_{\alpha}\right) \rho_{14}\left(\mathbf{r}_{14}\right) \cdot v_{\mathrm{NN}}^{\mathrm{DDM} 3 \mathrm{Y}}(s, \rho) \mathrm{d} \mathbf{r}_{\alpha} \mathrm{d} \mathbf{r}_{14}
$$

with $s=\left|\mathbf{r}_{14}-\mathbf{r}_{\alpha}-\mathbf{R}\right|$. Here $\mathbf{r}_{\alpha}\left(\mathbf{r}_{14}\right)$ denotes a coordinate measured from the center of mass in $\alpha\left({ }^{14} \mathrm{C}\right)$. $\rho_{\alpha}\left(\mathbf{r}_{\alpha}\right)$ denotes the density of $\alpha$ particle, which reproduce the charge form factor of the electron scattering, while $\rho_{14}\left(\mathbf{r}_{14}\right)$ represents the density of ${ }^{14} \mathrm{C}$, which is calculated by the Harmonic Oscillator (HO) model. The HO density of ${ }^{14} \mathrm{C}$ is designed to reproduce the observed matter radius, $r_{\mathrm{rms}}=2.30 \mathrm{fm}$, which is obtained from the analysis of the interaction cross section [14].

In Eq.(1), $v_{\mathrm{NN}}^{\mathrm{DDM}}$ Y represents the effective nucleonnucleon $(\mathrm{NN})$ interaction which acts between a pair of nucleon contained in the $\alpha$ particle and the ${ }^{14} \mathrm{C}$ nucleus. In the present calculation, we adopt the DDM3Y (Density Dependent Michigan 3-range Yukawa) interaction [1517], which is given by the functional form of

$$
v_{\mathrm{NN}}^{\mathrm{DDM} 3 \mathrm{Y}}(s, \rho)=v_{\mathrm{NN}}^{\mathrm{M} 3 \mathrm{Y}}(s) \cdot C\{1+\alpha \exp [-\beta \rho]\},
$$

where $v_{\mathrm{NN}}^{\mathrm{M} 3 \mathrm{Y}}$ represents the Michigan 3-range Yukawa interaction [18]. The factors of $C, \alpha$ and $\beta$ depend on the incident energy per nucleon, and they are determined so as to reproduce the strength of the nucleon potential inside of the nuclear matter [15]. In the previous application of DDM3Y, the so-called frozen density approximation (FDA), $\rho=\rho_{\alpha}+\rho_{14 \mathrm{C}}$, is employed [16]. However, this approximation is known to give a little shallow potential in the case of the $\alpha$ scattering [17]. Thus, we neglect the $\alpha$ density, $\rho_{\alpha}$, according to the recent prescription called the target density approximation (TDA) [19].

In the realistic calculation of the scattering cross sections, we introduce the absorptive potential $(-i W)$ and the 
Coulomb potential $\left(V_{\mathrm{C}}\right)$ in addition to the DF potential. The final form of the interaction potential $\left(U_{\text {int. }}\right)$ is

$$
U_{\text {int. }}=N_{\mathrm{r}} \cdot U_{\mathrm{DF}}+V_{\mathrm{C}}-i W
$$

Here we use the Saxon-Woods and its derivative form factor for the absorptive potential $(W)$. In this interaction potential, we multiply the normalization factor of $N_{\mathrm{r}}$ to the DF potential because there is theoretical ambiguity in the strength of the DF potential. As for the Coulomb potential $\left(V_{\mathrm{C}}\right)$, we assume the uniform charge sphere with a radius of $R_{\mathrm{C}}=r_{0}\left(4^{1 / 3}+14^{1 / 3}\right)$. The six parameters in SaxonWoods (depth, radius, diffuseness in each form factor) and $N_{\mathrm{r}}$ are tuned so as to reproduce the experimental cross sections as much as possible.

\subsection{Orthogonality condition model}

We calculate the energy spectra of ${ }^{18} \mathrm{O}=\alpha+{ }^{14} \mathrm{C}$ by employing the DF potential. In the energy calculation of the compound system, it is quite important to take into account the Pauli's exclusion principle between the $\alpha$ particle and ${ }^{14} \mathrm{C}$ nucleus. In the cluster systems, some spurious states, which is forbidden by the exclusion principle, arise in a simple potential model and hence, the forbidden states must be excluded from the computational space. The construction of the allowed functional space can be achieved by the orthogonality condition model (OCM) [12].

In OCM, the functional space that satisfies the exclusion principle can be produced by solving the eigenvalue problem of the norm kernel, which is symbolically given by

$$
\int \mathrm{d} \mathbf{r}^{\prime} \hat{N}\left(\mathbf{r}, \mathbf{r}^{\prime}\right) \chi_{\mu}\left(\mathbf{r}^{\prime}\right)=\lambda_{\mu} \chi_{\mu}(\mathbf{r})
$$

with the $\mu$-th norm eigenvalue of $\lambda_{\mu}$ and the corresponding relative wave function of $\chi_{\mu}$. The solutions with $\lambda \neq 0$ are the Pauli allowed states for the $\alpha-{ }^{14} \mathrm{C}$ relative motion, which should be included in the computational space, while the solution of $\lambda=0$ are excluded. The explicit expression of the norm kernel $\hat{N}\left(\mathbf{r}, \mathbf{r}^{\prime}\right)$ is

$$
\hat{N}\left(\mathbf{r}, \mathbf{r}^{\prime}\right)=\left\langle\delta(\mathbf{r}-\mathbf{a}) \varphi_{\alpha} \phi_{14} \mid \mathcal{A}\left\{\delta\left(\mathbf{r}^{\prime}-\mathbf{b}\right) \varphi_{\alpha} \phi_{14}\right\}\right\rangle,
$$

where $\varphi_{\alpha}$ and $\phi_{14}$ are the internal wave functions of $\alpha$ and ${ }^{14} \mathrm{C}$, respectively. The intrinsic structure of the $\alpha$ particle is set to $\left(0 s_{1 / 2}\right)^{4}$, while $(0,2)$ representation in $\mathrm{SU}(3)$ is used for ${ }^{14} \mathrm{C}$, which is projected to the $J^{\pi}=0^{+}$state. In Eq. (5), the bra-ket means the integration over the valuables of $\mathbf{a}$, b and all the internal coordinate contained in $\alpha$ and ${ }^{14} \mathrm{C}$.

In the previous studies, the wave function of $\chi_{\mu}$ is expanded in terms of the Harmonic Oscillator (HO) basis, and the matrix elements of the norm kernel is derived according to the mathematical technique in RGM (resonating group method) [20]. However, the analytic treatment in RGM is quite tedious and complicated for general $N \neq Z$ systems. Therefore, we handle the norm kernel in a way of the numerical calculation based on the prescription of GCM (generator coordinate method) [20].

Here we briefly explain the computational step of the numerical treatment; first, we solve a simple HO problem with the shifted Gaussian basis, which is a basis function of the GCM norm kernel. Secondly, the GCM norm kernels corresponding to the shifted Gaussian used in solving HO problem are calculated. Finally, the GCM norm kernels are transformed into the matrix element of the norm kernels of the HO basis. In this computational process, only the GCM norm kernels, which are easily calculated in a numerical manner, is used to evaluate the matrix elements of the norm kernel in Eq. (5) with the HO basis. The details of the computational procedure will be published in a forthcoming article.

\subsection{OCM under the absorbing boundary condition}

After the construction of the Pauli allowed states, the $\alpha-$ ${ }^{14} \mathrm{C}$ relative wave function $(\Psi)$ is expanded in terms of the Pauli allowed state, such as

$$
\Psi=\sum_{\mu}^{\prime} d_{\mu} \cdot \chi_{\mu},
$$

where the prime means that only the allowed states with $\lambda_{\mu} \neq 0$ is included in the summation. The energy spectra are calculated by solving the OCM equation, which is given by

$$
\left(T+N_{\mathrm{r}} \cdot U_{\mathrm{DF}}+V_{\mathrm{C}}-i \eta \tilde{W}-E\right) \sqrt{\hat{N}} \Psi=0
$$

with the expansion of Eq. (6). In Eq. (7), $T, U_{\mathrm{DF}}, V_{\mathrm{C}}$ denote the kinetic energy, the double folding potential, the Coulomb potential, respectively, while $E$ and $\hat{N}$ are the energy eigenvalues, and the norm kernels, respectively. The normalization factor of $N_{\mathrm{r}}$, which is fixed in the scattering calculation, is also used for the structure calculation, and only the real part of the interaction potential in Eq. (3) is included in the OCM equation.

In Eq. (7), $-i \eta \tilde{W}$ denotes the absorptive potential with the strength of $\eta$ but it differs from the absorptive potential using in the scattering calculation. This absorptive potential is introduced to impose the absorbing boundary condition (ABC) [21] to identify the resonant states, which appears above the $\alpha$ threshold. In the present calculation, we employ the shifted linear function with a step function $\theta(x)$ like

$$
\tilde{W}(r)=\theta\left(r-r_{a}\right)\left(r-r_{a}\right) .
$$

Here $r_{a}$ represents a starting point of the absorber, which is set to $r_{a}=8.0 \mathrm{fm}$ in the present calculation.

\section{Results}

\subsection{Analysis of $\alpha+{ }^{14} \mathrm{C}$ elastic scattering}

From the comparison of the theoretical calculation with the experiment, we check the validity of the DF potential with TDA. Comparison of the scattering calculations with the observed differential cross sections is shown in Fig. 1. The scattering calculation is done by the computational code of Automatic Local Potential Search (ALPS) [22]. We have calculated the differential cross section in the energy range from $E_{\alpha} / A_{\alpha}=5.5 \mathrm{MeV}$ to $E_{\alpha} / A_{\alpha}=8.8 \mathrm{MeV}$. 


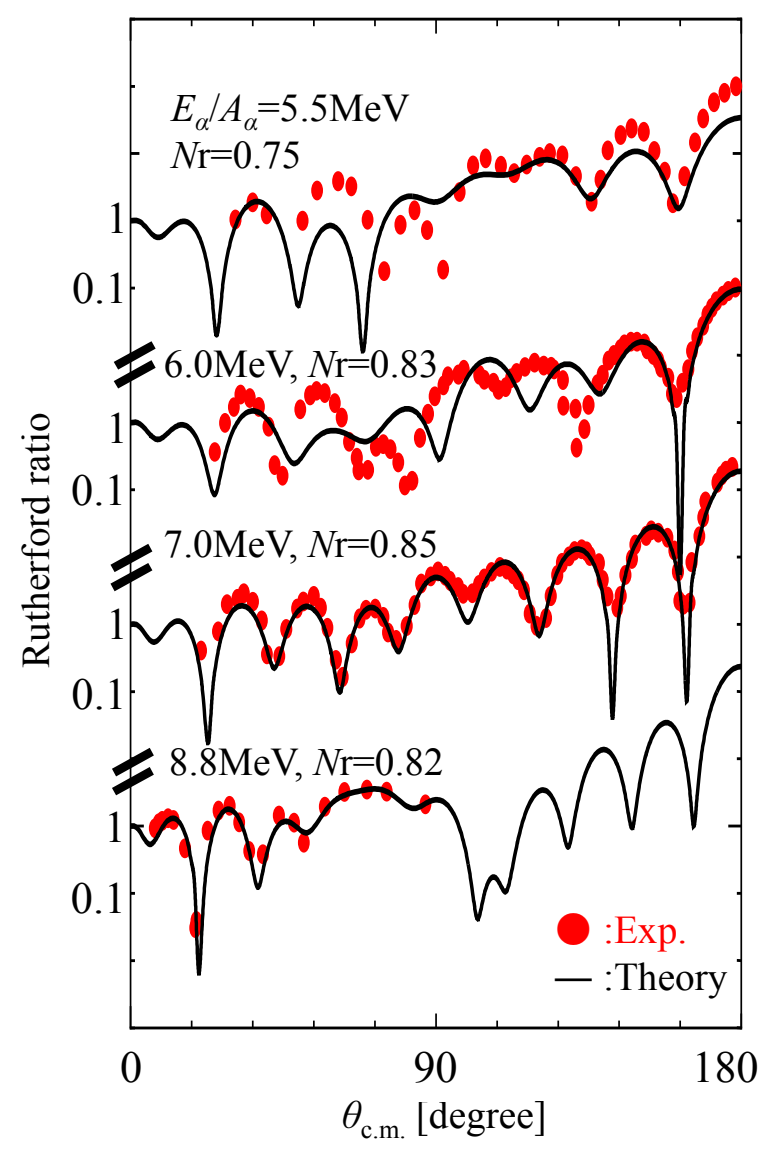

Figure 1. Compasiron of the theory and expermental data concering the $\alpha+{ }^{14} \mathrm{C}$ elastic scattering. The theoretical calculations and the observed differential cross sections are plotted by the solid curves and the solid circles, respectively. The abscissa shows the scattering angle in the center of mass system, while the ordinate represents the Rutherford ratio. The $N_{\mathrm{r}}$ attached to the individual comparisons represents the normalization factor multiplied to the DF potential.

The theoretical calculation (solid curves in Fig. 1), reasonably reproduce the observed cross sections (solid circles) over a whole range of the collision energies. The parameters of the absorptive potential are energy dependent, while the normalization factors of the DF potential are almost the energy independent, say about $N_{\mathrm{r}} \sim 0.8$. The reasonable reproductions of the observed cross section suggests that the employed DF potential with the TDA treatment is reliable interaction to describe the elastic scattering.

\subsection{OCM + ABC calculation for ${ }^{18} \mathrm{O}=\alpha+{ }^{14} \mathrm{C}$}

We solve the $\mathrm{OCM}+\mathrm{ABC}$ equation, which is given in Eq. (7), for the ${ }^{18} \mathrm{O}=\alpha+{ }^{14} \mathrm{C}$ cluster system by employing the DF potential used in the elastic scattering at $E_{\alpha} / A_{\alpha}=5.5 \mathrm{MeV}$. The $0^{+}$energy spectra plotted in a complex energy plane are shown in Fig. 2. In the case without absorbing potential, which is a calculation of $\eta=0$, all the energy levels appears along the real axis (solid circles). This $\eta=0$ calculation just corresponds to the simple bound-state-approximation. On the contrary, a series of the energy spectra are distributed in a complex energy plane (open circles) when the absorbing potential is switched on $(\eta=0.3)$ [21]. There are two kinds of the split continuum levels with a boundary of the sequence of the eigenvalues parallel to the real axis. The levels above the parallel line are the converged continuum state, in which the tail part of their wave function is smoothly damped in the asymptotic region, while the levels below the parallel line are the diverging state, where the wave function is artificially damped due to the finite range of the employed basis functions. On the contrary, the levels forming the line parallel to the real axis are called the indifferent state, and the wave functions in these states have a strong oscillating behavior over a whole spatial range.

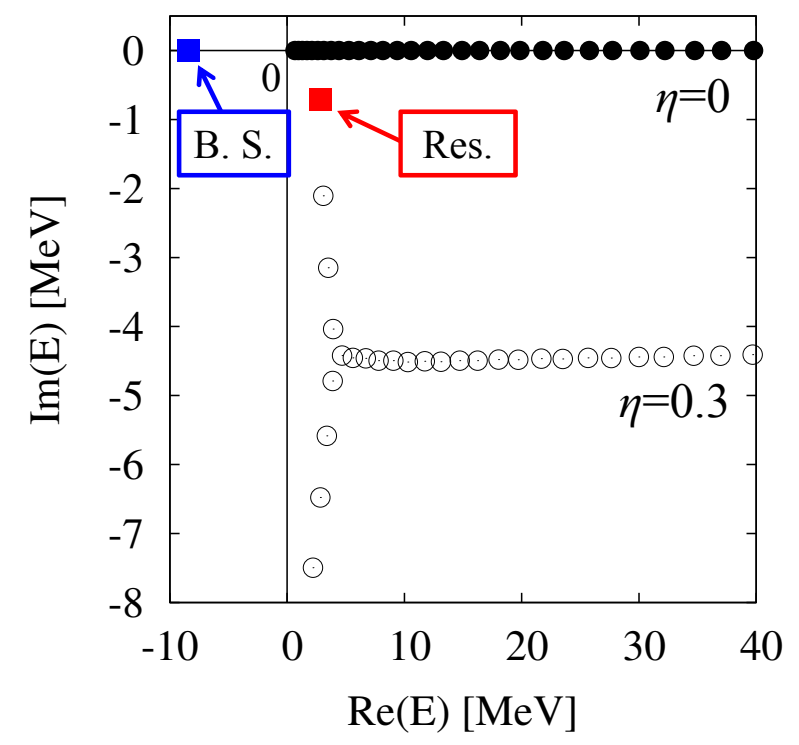

Figure 2. Complex energy spectra calculated from $\mathrm{OCM}+\mathrm{ABC}$ in Eq. (7). The abscissa and ordinate represent the real and imaginary parts of the energy eigenvalues. The zero energy in the real axis is set to the $\alpha$ decay threshold. The spectra on the real axis (solid circles) are the solution without the absorber $(\eta=0)$, while those distributed in the complex energy plane (solid squares and open circles) are the results with the abosrbing potential $(\eta=0.3)$. The solid squares show the bound state (B.S.) and the resonant state (Res.), while the open circles mean the non-resonant continuum states.

In the finite absorber calculation $(\eta=0.3)$, the bound state (B.S.) and the resonant state (Res.), which are plotted by the solid square are clearly separated from the nonresonant continuum state (open circles). The energy of the bound state is $E \sim-10 \mathrm{MeV}$ with respect to the $\alpha$ threshold, while the resonance energy is $E_{\mathrm{R}} \sim 3 \mathrm{MeV}$. The theoretical binding energy of the ground $0_{1}^{+}$state is over bound in comparison to the respective ground state in the experiment $\left(E_{\text {exp. }}=-6.2 \mathrm{MeV}\right)$. The absolute value of the binding energy is sensitive to the computational setting of the basis function in solving the eigenvalue problem of the norm kernel in Eq. (4). We have checked the several parameter sets of the shifted Gaussian in calculateing the GCM kernels and found the total binding energy considerably depends on the employed parameter sets, such as the 
width parameter or the mesh of the distant parameter in the shifted Gaussian. The basis dependence of the calculated energy will be discussed in future studies. However, the over binding feature of the ground state is invariant even if we change the parameter set of the shifted Gaussian. On the contrary, the energy of the excited $0_{2}^{+}$state $\left(E_{\mathrm{R}} \sim 3\right.$ $\mathrm{MeV}$ ) obtained in the present calculation is not so sensitive to the choice of the parameter set in the shifted Gaussian. The energy of the calculated $0_{2}^{+}$state seems to correspond to the $0_{4}^{+}$state, which is assigned in the recent experiment [23] although the resonance energy is a little higher than the experimental observation $\left(E_{\text {exp. }}=1.6 \mathrm{MeV}\right)$.

The deep binding feature of $0_{1}^{+}$is originated from the depth of the DF potential, which depends on the incident energy of the $\alpha$ particle. The parameters in DDM3Y ( $C$, $\alpha, \beta$ in Eq. (2)) are energy dependent, and the DF potential gets deeper and deeper as the incident energy becomes the lower. In the present $\mathrm{OCM}+\mathrm{ABC}$ calculation, we use the DF potential at the lowest energy, $E_{\alpha} / A_{\alpha}=5.5 \mathrm{MeV}$ and hence, this incident energy gives the deeper potential than the realistic potential, which should give the correct binding energy. In order to eliminate the energy dependence, we need to average of the DF potential over a finite range of the $\alpha$ incident energies. The averaged potential is expected to improve the over bound feature of the ground $0_{1}^{+}$ state.

In contrast to the $0_{1}^{+}$state, the excited $0_{2}^{+}$state in theory is expected to be insensitive to the depth of the DF potential because this resonance has the well developed $\alpha+{ }^{14} \mathrm{C}$ structure, in which the main amplitude of the relative wave function is concentrated around the tail part of the nuclear potential. Once the $\alpha$ cluster structure is strongly developed, the relative wave function of $\alpha-{ }^{14} \mathrm{C}$ becomes the higher nodal state involving a prominent extension. Thus, the relative wave function mainly feels the kinetic energy and the Coulomb interaction, and the nuclear interaction is not so effective in the cluster state.

\subsection{Coulomb shift in ${ }^{18} \mathrm{O}-{ }^{18} \mathrm{Ne}$}

We have performed the similar $\mathrm{OCM}+\mathrm{ABC}$ calculation for $\alpha+{ }^{14} \mathrm{O}$, and the $0^{+}$energies are calculated for the ${ }^{18} \mathrm{Ne}$ nucleus. In this calculation, the nuclear potential of the $\alpha+{ }^{14} \mathrm{O}$ system is set to the same DF potential as that used in the $\alpha+{ }^{14} \mathrm{C}$ calculation, and only the charge number in the Coulomb potential of $V_{\mathrm{C}}$ in Eq. (7) is changed. The energy shift of ${ }^{18} \mathrm{O}-{ }^{18} \mathrm{Ne}$, which is defined by $\Delta E=\left|E\left({ }^{18} \mathrm{O}\right)-E\left({ }^{18} \mathrm{Ne}\right)\right|$, is summairzed in table. 1 .

Table 1. Energy shift $\Delta E=\left|E\left({ }^{18} \mathrm{O}\right)-E\left({ }^{18} \mathrm{Ne}\right)\right|$ for the $0^{+}$state. The energy shift for the ground $0_{1}^{+}$state is shown in the upper row, while the bottom row shows the energy shift for the excited $0^{+}$state.

\begin{tabular}{c||cc}
\hline & Exp. [MeV] & Theory $[\mathrm{MeV}]$ \\
\hline$\Delta E\left(\right.$ Ground $\left.0^{+}\right)$ & 1.1 & 1.6 \\
\hline$\Delta E\left(\right.$ Excited $\left.0^{+}\right)$ & - & 0.8 \\
\hline
\end{tabular}

The energy shift of ${ }^{18} \mathrm{O}-{ }^{18} \mathrm{Ne}$ is insensitive to the choice of the parameter set in the shifted Gaussian because the variation of the energies with respect to the change of the basis set is common in these two systems. Theoretical energy shift of the $0_{1}^{+}$state, which is shown in the upper row, is about $\Delta E\left(0_{1}^{+}\right)=1.6 \mathrm{MeV}$. This energy shift is a little larger than the experimental observation of $\Delta E\left(0_{1}^{+}\right)=1.1 \mathrm{MeV}$. This overestimation of the present calculation is due to the deep binding feature of the $0_{1}^{+}$energies, which is calculated from the DF potential at the lowest $\alpha$ scattering energy. Thus, the energy average of the DF potential should be taken into account in the evaluation of the Coulomb shift of the ground state. On the contrary, the theoretical calculation predicts that the Coulomb shift for the excited $0^{+}$state is about $\Delta E\left(0_{2}^{+}\right)=0.8 \mathrm{MeV}$, which is reduced to be about half the ground shift of $\Delta E\left(0_{1}^{+}\right)=1.6$ $\mathrm{MeV}$. There is no experimental data of the energy shift, which is evaluated by $\Delta E=\left|E\left({ }^{18} \mathrm{O}\left(0_{4}^{+}\right)\right)-E\left({ }^{18} \mathrm{Ne}\left(0_{4}^{+}\right)\right)\right|$. This is due to the lack of the experimental information of the resonant levels in ${ }^{18} \mathrm{Ne}$ above the $\alpha+{ }^{14} \mathrm{O}$ threshold.

The reduction of the energy shift for the excited $0_{2}^{+}$ state is induced by the development of the $\alpha$ clustering. If the compound system is excited to the cluster state, the spatial distance between the $\alpha$ particle and the residual nucleus $\left({ }^{14} \mathrm{C}\right.$ or $\left.{ }^{14} \mathrm{O}\right)$ is prominently extended. In fact, we have checked the relative wave functions of the ground $0_{1}^{+}$ state and the excited $0_{2}^{+}$state and confirmed the root-meansquare (r.m.s.) radius of the wave function in the cluster $0_{2}^{+}$state is enhanced by about $3.5 \mathrm{fm}$ in comparison to the r.m.s. radius of the $0_{1}^{+}$wave function. Therefore, the expectation value of the Coulomb interactions in the cluster state is weaker than the expectation value in the ground state, in which $\alpha$ and the residual nucleus is strongly overlapped each other. This suppression of the Coulomb effect leads to the reduction of the energy shift in the mirror systems, which can be clearly confirmed in table. 1 . The present results strongly suggests that the reduced Coulomb shift is a new probe to identify the cluster degrees of freedom, which appears in the low excited state.

\section{Summary and discussion}

In summary, we have performed the scattering and structure calculations for the ${ }^{18} \mathrm{O}=\alpha+{ }^{14} \mathrm{C}$ system. In the scattering calculations, the $\alpha+{ }^{14} \mathrm{C}$ interaction potential is constructed from the double folding (DF) model, which employs the effective nucleon-nucleon interaction of the density-dependent Michigan 3-range Yukawa (DDM3Y). In the derivation of the DF potential, only the density dependence of ${ }^{14} \mathrm{C}$ is considered in accordance to the recent prescription of target density approximation (TDA). The DF potential plus the absorptive potentials, which are determined in a phenomenological manner, reasonably reproduces the observed differential cross section of the $\alpha+{ }^{14} \mathrm{C}$ elastic scattering.

The DF potential is applied to the structure calculation based on the orthogonality condition model (OCM) under the absorbing boundary condition (ABC). In the previous applications of OCM, the norm kernel is evaluated analytically by employing the sophisticated technique of the resonating group method (RGM). Since the evaluation of the RGM norm kernels is quite tedious and complicated, 
we have formulated the simple computational method of the norm kernel on the basis of the generator coordinate method (GCM). In this treatment, only the GCM norm kernels, which can be evaluated from the numerical calculation, is used in handling the eigenvalue problem in OCM.

In the $\mathrm{ABC}+\mathrm{OCM}$ calculation, we have obtained the two $0^{+}$states: the ground $0_{1}^{+}$state and the excited $0_{2}^{+}$state. The binding energy of the ground $0_{1}^{+}$state is a little over bound but the excitation energy of the excited $0_{2}^{+}$state seems to be coincident to the $0_{4}^{+}$state, which has been confirmed in the recent experiment. In order to improve the over binding problem of the ground state, we need to consider the energy average of the DF potential, which depends on the incident energy of the $\alpha$ particle. The energy average of the DF potential is now considering.

We have also performed the similar structure calculation for the mirror partner, the ${ }^{18} \mathrm{Ne}=\alpha+{ }^{14} \mathrm{O}$ system, by replacing the Coulomb potential of ${ }^{18} \mathrm{O}$ to that of ${ }^{18} \mathrm{Ne}$. We compare the energy shift of these mirror systems, which is mainly originated from the difference of the Coulomb interaction. From this comparison, we have confirmed that the Coulomb shift of the excited $0_{2}^{+}$is prominently reduced in comparison to the shift of the ground $0_{1}^{+}$state. This difference of the Coulomb shift has a close connection to the intrinsic structure in the individual $0^{+}$states: the developed $\alpha$ cluster structure in the excited $0_{2}^{+}$state and the melted cluster structure in the ground $0_{1}^{+}$state. In the excited state, the spatial extension is prominent due to the $\alpha$ cluster formation, and this extension leads to the suppression of the Coulomb interaction between $\alpha$ and the residual nuclei $\left({ }^{14} \mathrm{O}\right.$ and $\left.{ }^{14} \mathrm{C}\right)$. On the contrary, in the ground state, the $\alpha$ particle and ${ }^{14} \mathrm{C}$ are strongly overlapped each other and hence, the Coulomb interaction is strong. Therefore, the Coulomb shift of the excited state is strongly suppressed in comparison to the ground state due to the spatial extension of the $\alpha$ cluster.

The present result strongly suggests a new possibility of the Coulomb shift as a probe of the cluster degrees of freedom. However, the present calculation is still insufficient to discuss the realistic Coulomb shift observed in the experiment because the present calculation does not reproduce the low-lying bound $0^{+}$states below the $\alpha$ threshold. In ${ }^{18} \mathrm{O}$, for example, the $0_{2}^{+}$and $0_{3}^{+}$states appear at the excitation energy of $3.63 \mathrm{MeV}$ and $5.34 \mathrm{MeV}$, respectively. These bound state cannot be reproduced by the present calculation, which includes the single cluster configuration of $\alpha+{ }^{14} \mathrm{C}$. Thus, we introduce other configurations to reproduce the bound levels. In order to improve the calculation, we need to include the two particle configuration around ${ }^{16} \mathrm{O}$ core and the excitation of ${ }^{14} \mathrm{C}$ in the $\alpha+{ }^{14} \mathrm{C}$ configuration. As demonstrated in Ref. [24], the two particle configuration is essential to reproduce the bound $0^{+}$states, while the excitation of ${ }^{14} \mathrm{C}$ is expected to be important for the formation of the higher resonances. The extended calculation taking into account these configurations are now underway.
In addition to the theoretical improvement, the experimental investigation of ${ }^{18} \mathrm{Ne}$ is strongly desired. In the bound region, the $0^{+}$levels corresponding to those in ${ }^{18} \mathrm{O}$ have already been identified but the identification of the resonant levels above the $\alpha$ decay threshold is still insufficient in ${ }^{18} \mathrm{Ne}$. In particular, the experimental investigation of the resonant $0^{+}$state is very interesting because the cluster degrees of freedom will be strongly activated. The development from both of the theory and experiment will be important subject in future studies.

\section{References}

[1] H. Horiuchi et al., Suppl. Prog. Theor. Phys. 192, 1 (2012), and references therein.

[2] A. Tohsaki, H. Horiuchi, P. Schuck and G. Reöpke, Phys. Rev. Lett. 87, 192501 (2001).

[3] Y. Funaki et al., Phys. Rev. Lett. 101, 082502 (2008)

[4] Y. Funaki, H. Horiuchi, W. von Oertzen, G. Reöpke, P. Schuck, A. Tohsaki and T. Yamada, Phys. Rev. C 80, 064326 (2009).

[5] M. Ito and K. Ikeda, Rep. Prog. Phys. 77, 096301 (2014), and references therein.

[6] N. Itagaki, T. Otsuka, K. Ikeda, and S. Okabe, Phys. Rev. Lett. 92, 142501 (2004).

[7] M. Kimura, Phys. Rev. C 75, 034312 (2007).

[8] A. Bohr and B. R. Mottelson, Nuclear Structure vol. I (New York: Benjamin, 1969) p.320.

[9] M. Ito, EPJ Web of conf. 122 (2016).

[10] P. Descouvemont and D. Baye, Phys. Rev. C 31, 2274 (1985).

[11] N. Furutachi et al., Prog. Theor. Phys. 119, 403 (2008).

[12] A. Saito, Suppl. Prog. Theor. Phys. 62, 11 (1977).

[13] M. Ito, Y. Hirabayashi and Y. Sakuragi, Phys. Rev. C 66, 034307 (2002), and references therein.

[14] A. Ozawa, T. Suzuki and I. Tanihata, Nucl. Phys. A693, 32 (2001).

[15] A. M. Kobos et al., Nucl. Phys. A384 (1982); A. M. Kobos, B. A. Brown, R. Lindsay and G. R. Satchler, Nucl. Phys. A425, 205 (1984).

[16] M. El-Azab Farid and G. R. Satchler, Nucl. Phys. A438, 525 (1985).

[17] M. E. Brandan and G. R. Satchler, Nucl. Phys. A487, 477 (1988).

[18] G. R. Satchler and W. G. Love, Phys. Rep. 55 (1979).

[19] K. Egashira et al., Phys. Rev. C 89,064611 (2014).

[20] H. Horiuchi, Suppl. Prog. Theor. Phys. 62, 90 (1977).

[21] M. Iwasaki, R. Otani, Y. Takenaka and M. Ito, PTEP 2015, $023 \mathrm{D} 01$.

[22] Y. Iseri (private communication).

[23] W. von Oertzen et al., Eur. Phys. J. A 43, 17 (2010).

[24] H. Furutani et al., Suppl. Prog. Theor. Phys. 68, 279 (1980). 